\title{
Voltage Quality Analysis of a Three-Level Flying Capacitor Inverter with Model Based Predictive Control
}

\author{
S. Thielemans, T.J. Vyncke, J.A.A. Melkebeek \\ Department of Electrical Energy, Systems and Automation (EESA) \\ Ghent University (UGent), Sint-Pietersnieuwstraat 41, B-9000 Gent, Belgium \\ Steven.Thielemans@UGent.be; Thomas.Vyncke@UGent.be; Jan.Melkebeek@UGent.be
}

\begin{abstract}
Model based predictive control is an interesting control method for power electronics as it offers many advantages. A known disadvantage compared to optimal pulse-width modulation control is the deteriorated voltage quality, which results in extra losses in the load. In this paper two ways of analyzing the output voltage quality are presented and applied on the model based predictive control of a three-level flying capacitor inverter. The influence of the weight factor and the system model are analyzed. The voltage quality is shown to be good in the same weight factor range as those resulting in good current and capacitor voltage control. No compromises have to be made in this area. A more accurate system model results in improved voltage (and overall control) quality.
\end{abstract}

Index Terms-Motor Control and Drives, Other Areas in Power Electronics

\section{INTRODUCTION}

Multilevel converters have several advantages compared to two-level converters. The intermediate output voltage levels result in lower harmonics in the output current. Besides the better output quality, also a higher voltage handling is possible due to a series connection of switch components. The flying capacitor (FC) converter topology has recently attracted a lot of interest in literature, [1]-[13], because of the advantages over other multilevel topologies (e.g. neutral-point-clamped or cascaded converters), [3], [4].

An FC converter control needs a regulation of the capacitor voltages either passively by using the natural balancing property of a pulse width modulation (PWM) scheme, [10]-[13], or actively, [5]. It is shown in recent publications, [5], that a passive control can fail in certain circumstances. It is theoretically proven, [11], [12], that certain time constants of the FC converter system (with level count higher than five) become infinite for some specific modulation indices. Because of these problems there is a trend towards active control. This active control is usually implemented seperately, additionally to the current control of the converter although these kinds of multiple-input-multiple-output (MIMO) systems generally benefit from a true multivariable control. Furthermore the switch state of the FC converter is inherently discrete. Finite set model based predictive control (MBPC) is by nature a MIMO and discrete control and is for these reasons increasingly used in power electronics applications, [14]-[17].

In previous publications the control quality of the output current and of the capacitor voltages using MBPC applied on FC converters is analysed, [18]. In this paper the output voltage quality will be analysed, especially the voltage quality as a function of the predictive control parameters in the ranges that are optimal for current and capacitor voltage control.

\section{Flying-CAPACITOR CONVERTERS MultileVEl TOPOLOGY}

The topology of the three-level FC inverter, discussed in this paper, is depicted in Fig. 1. Because a three-level FC inverter is the least complicated one with only one flying capacitor per phase, it is selected for a clear analysis of the MBPC. The threelevel FC converter uses 2 pairs of complementary controlled switches, $\left(S_{1}, \overline{S_{1}}\right)$ and $\left(S_{2}, \overline{S_{2}}\right)$. These switches make it possible to connect the flying capacitors, $C_{1 x}(x=a, b, c)$, in series with the load. The load is here represented as an RL series connection, to simulate a simple inductive load.

An overview of the possible switch states and their resulting output voltage is given in table I. When the upper switch of the switch pair $i(i=1,2)$ is closed, $S_{i x}$ is 1 , when the lower switch is closed $S_{i x}$ is zero. When the flying capacitor is connected in series with the load, the voltage of the capacitor changes as the load current flows through the capacitor. The voltage of the flying capacitor $C_{1 x}$ in a threelevel converter should always be kept at $V_{\mathrm{DC}} / 2$. 

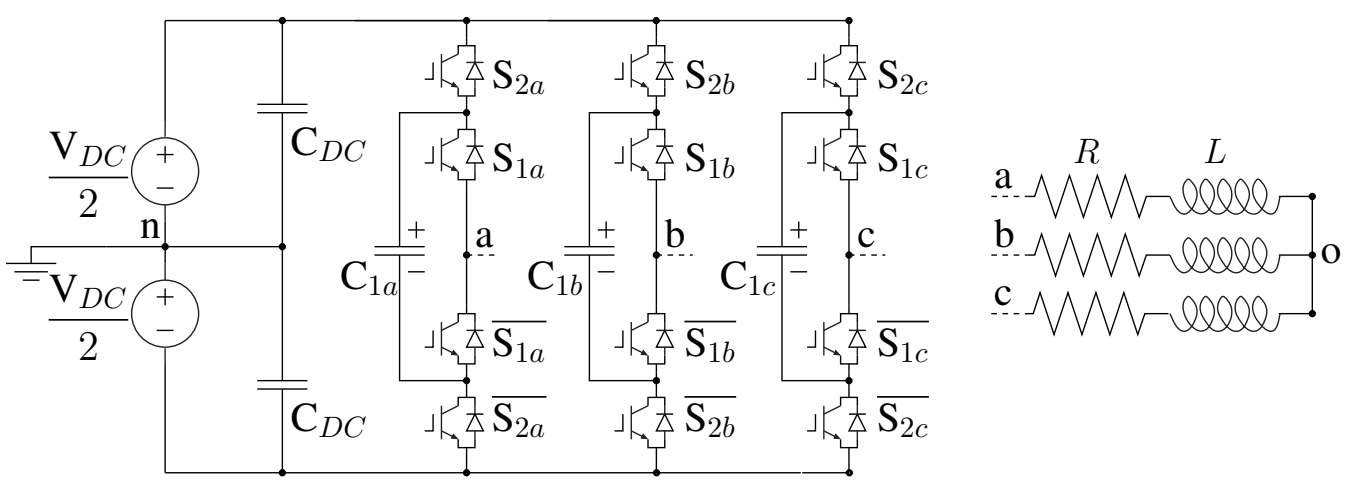

Figure 1. Three-level flying capacitor converter circuit topology, single phase leg.

Table I

SWITCH STATES AND THE CORRESPONDING OUTPUT VOLTAGE (WHEN $\left.V_{C_{1 x}}=V_{\mathrm{DC}} / 2\right)$

\begin{tabular}{c|cc|l}
$\#$ & $S_{1 x}$ & $S_{2 x}$ & $V_{x n}\left(V_{C_{x}}=V_{\mathrm{DC}} / 2\right)$ \\
\hline 1 & 0 & 0 & $-V_{\mathrm{DC}} / 2$ \\
2 & 1 & 0 & 0 \\
3 & 0 & 1 & 0 \\
4 & 1 & 1 & $V_{\mathrm{DC}} / 2$
\end{tabular}

This choice provides optimal voltage rating of the switches as the maximum voltage stress is $V_{\mathrm{DC}} / 2$. Each phase has two switch states which produce the intermediate output voltage. This makes it possible to do a correction of the capacitor voltage for every possible current direction. This gives us a means to control the capacitor voltage.

\section{Model Based Predictive Control for FC INVERTERS}

The main control objectives for MBPC with multilevel converters are the tracking of the reference current and the balancing of the flying capacitor voltages. Secondary control objectives are reducing the switching losses, harmonic elimination, EMI, etc. To this end the inputs for the MBPC algorithm are the reference values and the measurements of phase currents and flying capacitor voltages. The output of the algorithm is one of the possible switch states of the converter. As the switch state is maintained during an entire sample period, the possible outputs belong to a restricted set.

\section{A. Estimation Step}

At time instant $k$ a previously defined switch state is applied and measurements of the phase currents $\mathbf{i}_{x}(k)$ with $x=a, b, c$ and the flying capacitor voltages $\mathbf{v}_{c x}(k)$ are carried out. In the first step of
MBPC, the estimation step, the load currents and capacitor voltages at time instant $k+1$ are estimated based on a system model, the measurements at $k$ and applied control output at $k$. The known variables, either because they are selected (switch states) or because of measurements (current and voltage), are shown in bold characters. The system equations assume that the possible change in capacitor voltage is small and slow enough to neglect this change when calculating the output voltage. Under these assumptions the following expressions are obtained for the voltages $v_{x n}$ between the output pole $x$ and the neutral point $n$ of the power supply:

$$
v_{x n}^{k+1}=\left(\mathbf{S}_{2 x}^{k}-\frac{1}{2}\right) V_{\mathrm{DC}}-\left(\mathbf{S}_{2 x}^{k}-\mathbf{S}_{1 x}^{k}\right) \mathbf{v}_{c x}^{k},
$$

where the bus voltage $V_{\mathrm{DC}}$ is assumed known or measured. The load phase voltage $v_{x o}$, between the output pole and the load star-point $o$, is calculated with

$$
\begin{gathered}
v_{x o}^{k+1}=v_{x n}^{k+1}+v_{o n}^{k+1}, \\
v_{o n}^{k+1}=\frac{v_{a n}^{k+1}+v_{b n}^{k+1}+v_{c n}^{k+1}}{3}
\end{gathered}
$$

where $v_{o n}$ is the star-point voltage. The output voltage results in a current and capacitor voltage change:

$$
i_{x}^{k+1}=e^{-\Delta \frac{R}{L}} \mathbf{i}_{x}^{k}+\frac{1-e^{-\Delta \frac{R}{L}}}{R} v_{x o}^{k+1},
$$

with $R$ and $L$ the resistive and inductive parts of the load respectively and $\Delta$ is the update period;

$$
v_{c x}^{k+1}=\mathbf{v}_{c x}^{k}+\frac{\Delta}{2 C}\left(\mathbf{i}_{x}^{k}+i_{x}^{k+1}\right)\left(\mathbf{S}_{2 x}^{k}-\mathbf{S}_{1 x}^{k}\right)
$$

where $C$ is the capacitance value of the flying capacitor $C_{1 x}$. 


\section{B. Prediction Step}

From $k+1$ on the controller can use any possible output to bring the controlled variables closer to their desired values. In step two, the prediction step, the controller thus calculates the controlled variables at $k+2$ for all possible switch states at $k+1$. The output voltage is now:

$$
v_{x n}^{k+2}=\left(S_{2 x}^{k+1}-\frac{1}{2}\right) V_{\mathrm{DC}}-\left(S_{2 x}^{k+1}-S_{1 x}^{k+1}\right) v_{c x}^{k+1},
$$

which results in the phase load voltage by adding the star-point voltage:

$$
\begin{gathered}
v_{x o}^{k+2}=v_{x n}^{k+2}+v_{o n}^{k+2}, \\
v_{o n}^{k+2}=\frac{v_{a n}^{k+2}+v_{b n}^{k+2}+v_{c n}^{k+2}}{3} .
\end{gathered}
$$

This voltage can now be used to calculate the current:

$$
i_{x}^{k+2}=e^{-\Delta \frac{R}{L} \mathbf{i}_{x}^{k+1}}+\frac{1-e^{-\Delta \frac{R}{L}}}{R} v_{x o}^{k+2} .
$$

This current defines the capacitor voltage change if an intermediate voltage is selected:

$$
v_{c x}^{k+2}=\mathbf{v}_{c x}^{k+1}+\frac{\Delta}{2 C}\left(\mathbf{i}_{x}^{k+1}+i_{x}^{k+2}\right)\left(\mathbf{S}_{2 x}^{k+1}-\mathbf{S}_{1 x}^{k+1}\right) .
$$

The star-point voltage, (8), is the average voltage of the three-phases and this makes the solution for the three phases coupled with each other. In the prediction step, the model can be simplified by neglecting the influence of each phase on the other phases because of this star-point voltage $v_{o n}^{k+2}$. In case the star-point voltage is neglected, this is referred to as the uncoupled case, the normal unsimplified case is the coupled case. In the uncoupled case the star-point voltage $v_{o n}^{k+2}$ is zero in equation (7).

By neglecting this star-point voltage, the evaluation can be done for each phase separately (the uncoupled case), resulting in less possible switch states and less required processing power, [7], [18]. In the predictions step the uncoupled equations will of course result in inaccurate predictions because of the model simplification.

\section{Optimization Step}

In the third step, the optimization step, the optimal switch state is selected based on the minimum value of a cost function $g$ which expresses the deviation of the controlled variables from their desired values. This switch state is applied by the controller at time instant $k+1$ and at that time the total algorithm is started again from the measurements and estimation step (receding horizon). When using a quadratic cost function the converter phase cost function $g_{x}(k)$ can be defined as

$$
g_{x}^{k}=\left(i_{x, r}^{k+2}-i_{x}^{k+2}\right)^{2}+W_{v_{c}}\left(v_{c, r}^{k+2}-v_{c x}^{k+2}\right)^{2},
$$

where subscript $r$ means reference value. The weight factor $W_{v_{c}}$ expresses the relative importance of an error in the flying capacitor voltage compared to an current error. The total cost function $g^{k}$ is the sum of the phase cost functions.

$$
g^{k}=g_{a}^{k}+g_{b}^{k}+g_{c}^{k} .
$$

In the case where the star-point voltage is neglected, the problem can be solved by considering each phase cost function, selecting the minimum $g_{a}^{k}, g_{b}^{k}$ and $g_{c}^{k}$ separately.

\section{Optimal Control Quality Region}

In all predictive control methods, the controlled system variables correspond with a term in the cost function. In this application important controlled variables are the phase output currents and the three flying capacitor voltages. The weight factor, providing the relative importance of each term, should be chosen in a way that the requirements on the control variables are met. Because the system parameters of the model are never perfectly matched with the real system, a range for the weight factor to give good results is preferable. The control quality is evaluated using the Mean Square Error (MSE) value, see [18].

It was found that a good range for the weight factor $W_{v_{c}}$ can be found by evaluating the MSE of the current and the capacitor voltages in simulation. The system parameters for simulation are tabulated in table II. Figure 2 shows, as a function of weight factor $W_{v c}$, the control quality of the current (a), the capacitor voltage (b) and the sum of both (c) for a phase current amplitude of 4A. The width of the good weight factor range depends of the quality of the model. For example neglecting the star-point

Table II

TABLE WITH SYSTEM PARAMETERS USED IN SIMULATION.

\begin{tabular}{ll} 
Parameter & Value \\
\hline$V_{\mathrm{DC}}$ & $100 \mathrm{~V}$ \\
$L$ & $14.5 \mathrm{mH}$ \\
$R$ & $4.5 \Omega$ \\
$C$ & $110 \mu \mathrm{F}$ \\
$f_{u}$ & $20 \mathrm{kHz}$
\end{tabular}



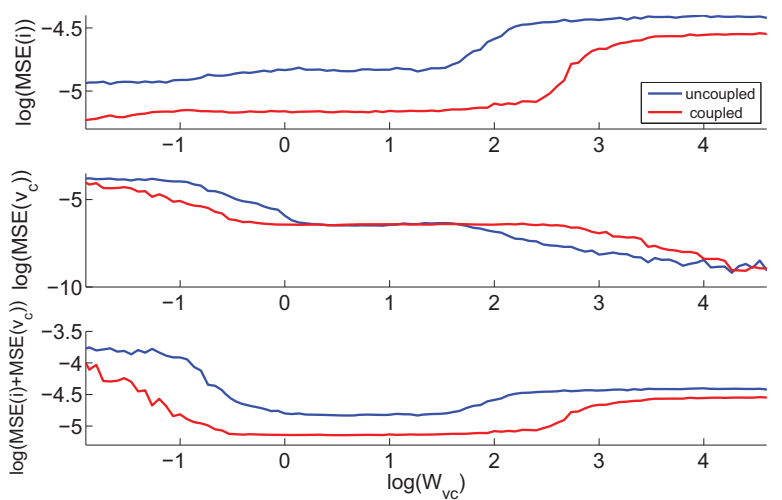

Figure 2. MSE as a function of $W_{v c}$ of (top) output current, (middle) capacitor voltage and (bottom) the sum of the MSE's of current and voltage to define overall control quality in simulation.

voltage, which is a drastic model simplification, will result in a worse control quality and in a much smaller range width. A wider range makes the selection of the weight factor less critical if the range position changes with the current amplitude. It results in easier selection of a weight factor for a broad current amplitude range and model imperfections.

The capacitor voltage control quality is similar in both cases, but the range is also wider in the coupled case. So from simulations the effect of the simplified model on the control quality is significant.

\section{Voltage Quality ANALYsis}

\section{A. Mean Square Error Method}

The useful voltage is the fundamental component of the output phase voltage. In an ideal case only the fundamental component is present, but using switching converters, harmonics are injected in the output voltage. The deviation of the instantaneous output voltage from the fundamental component results in poor voltage quality and can be assessed with the MSE value. The MSE is related to the RMS ripple in the output voltage. This way the MSE is also a measure for extra ohmic losses in the load. Figure 3 shows the output voltage MSE as a function of $W_{v c}$ for the coupled and uncoupled cases. The voltage quality for the coupled case is good and constant over the $W_{v c}$ range which results in a good current and capacitor voltage control quality. The voltage quality is significantly better in the coupled case than the uncoupled case. The range with good control is also wider for the coupled case.

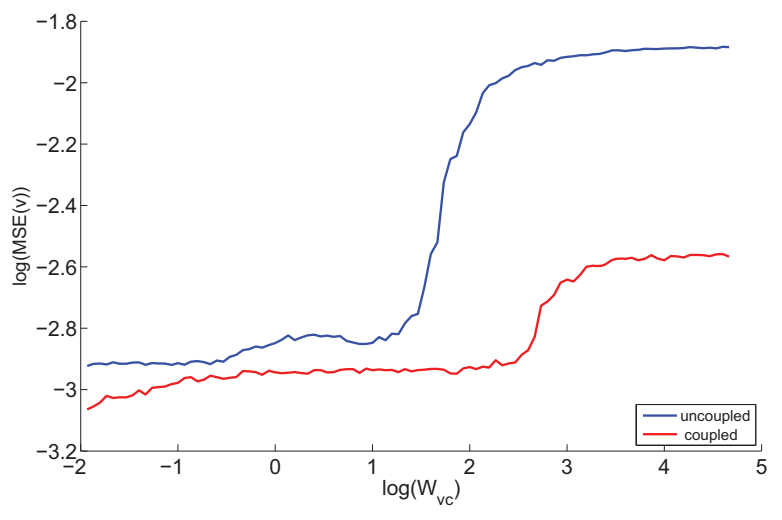

Figure 3. MSE of the output voltage as a function of $W_{v c}$ for MBPC on three-level FC inverter for the coupled case (red line, taking the star-point voltage into account) and the uncoupled case (blue line, neglecting star-point voltage).

\section{B. Three-phase State Transition}

The voltage quality can be also be assessed by analyzing the three-phase state transition. In [19], it is stated that an optimal control can only be achieved by selecting the nearest vector in the threephase space vector representation. The three-phase state transition analysis can be used to compare different modulation and control schemes. The threephase state transitions are analysed using the $(\alpha, \beta)$ representation of the voltages. These voltages can be calculated from the phase voltages using the Clarke transformation:

$$
\begin{aligned}
& v_{\alpha}=\frac{2}{3}\left(v_{a o}-\frac{v_{b o}}{2}-\frac{v_{c o}}{2}\right), \\
& v_{\beta}=\frac{1}{\sqrt{3}}\left(v_{b o}-v_{c o}\right) .
\end{aligned}
$$

Analyzing the distance between the consecutive voltage vectors results in the data depicted in figure 4. Here the dash-dot lines represent the relative number of updates without a change of the threephase voltage vector, both for the coupled $(\mathrm{C}$, red) and uncoupled (UC, blue) case. The dashed lines represent the updates with a change of only a single step (the smallest possible step) to the surrounding vectors. The sum of the case where no change happens or only a single step is represented by the full lines. The part of the switchings that is not included in this full line, is the part where a large step in the output voltage vector takes place. This should be avoided, as it doesn't make use of the full potential of the multilevel converter.

There is a very significant difference between the 


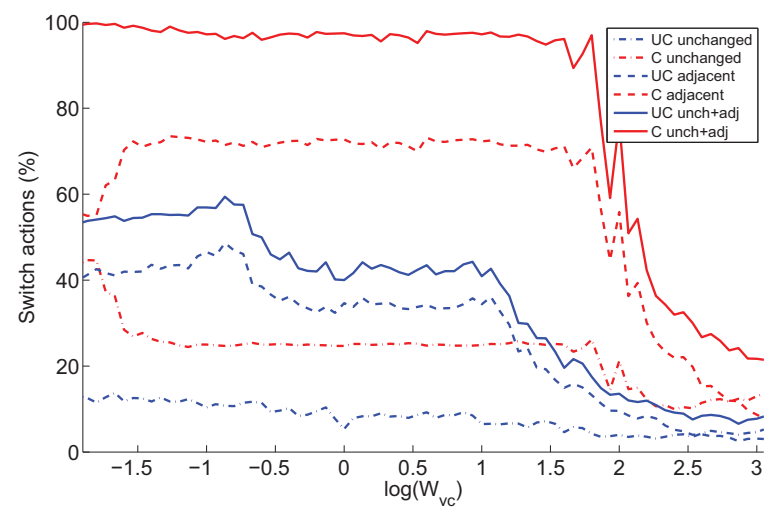

Figure 4. Nearest vector selection: full lines are the sum of the number of unchanged output vectors (dash-dot lines) and the number of transitions to adjacent vector selection (dashed lines) as a function of $W_{v c}$ for the coupled (red) and uncoupled (blue) case using simulation results.

coupled and uncoupled case. In the coupled case, over a wide range, the nearest vector criterion is more or less met, while for the uncoupled case, less than 60 percent of the updates is conform the nearest vector criterion. This large number of updates which result in large steps in the three-phase voltage is the cause of the high MSE value of the output voltage as depicted in figure 3.

For a high weight factor, the MBPC becomes reluctant to the use of the intermediate output phase voltage $\left(v_{x n}=0 \mathrm{~V}\right)$ because it influences the flying capacitor voltage. A small variation of the flying capacitor voltage generates a significant cost term in the cost function when the weight factor becomes large. A lot of vectors in the three-phase $\alpha / \beta$ diagram can only be reached using those intermediate phase voltages. For this reason it becomes more difficult to select a surrounding voltage vector for higher weight factors, as shown in figure 4 . These larger steps in the voltage are the cause of the sudden rise of the MSE value for higher weight factors.

\section{EXPERIMENTS}

\section{A. Experimental Setup}

The experimental setup is a three-level flying capacitor converter constructed from in-house, halfbridge power electronic building blocks (PEBB). Output current and flying capacitor voltage are measured in each phase leg and digitized with 12bit ADCs (Digilent PMOD AD1). Additionally the bus voltage $V_{\mathrm{DC}}$, supplied by a regulated source (Delta $300-10)$ is measured as well. The system parameters of the setup are the same as those used in the simulation study. The converter is controlled with an Xilinx VirtexII-Pro FPGA (XUPV2P-30), clocked at $100 \mathrm{MHz}$.

Both the decoupled and the coupled version (including the star-point voltage) are implemented in the FPGA in digital hardware. Configuration of the FPGA is done in VHDL and with the Xilinx Simulink tool System Generator. The computational load clearly is much higher in the coupled case, as there are 6 additional operations for each switch state possibility and 16 times as many input combinations have to be considered. Using an optimal implementation with pipe-lined operations, it is possible to obtain a cycle time of $6 \mu \mathrm{s}$, so the update frequency of $20 \mathrm{kHz}$ is easily achievable. Meanwhile only about one third of the FPGA slices and embedded multipliers are used for the coupled case.

\section{B. Experimental Results}

An oscilloscope image of a measurements of the FC inverter controlled with the proposed MBPC is shown in figure 5. The weight factor for this situation is $W_{v c}=10$, using the uncoupled case, the current amplitude is $4 \mathrm{~A}$ with a frequency of $50 \mathrm{~Hz}$. The quality of the current waveform is not perfect, mostly because of the uncoupled equations. In the phase voltage $v_{a o}$ depicted, it is possible to recognize the five levels created with the threelevel phase leg combined with the varying star-point voltage influenced by all three phase voltages.

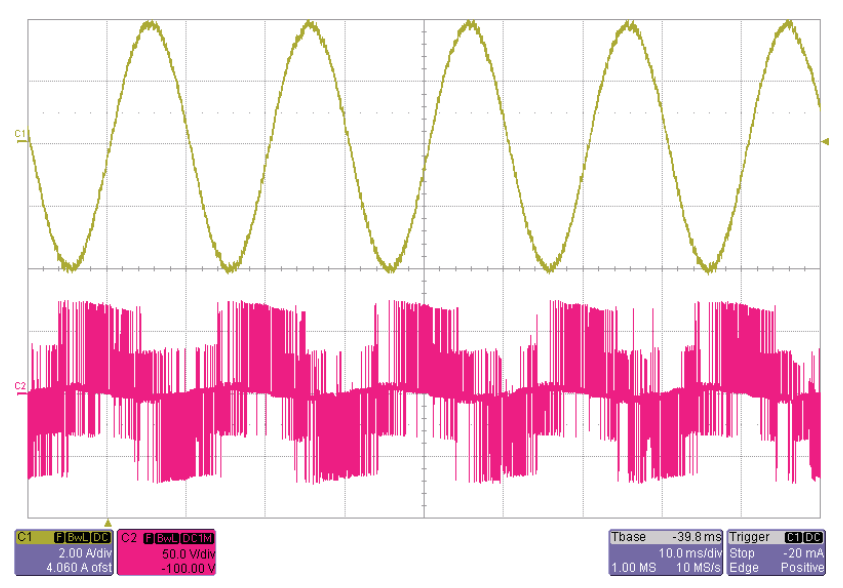

Figure 5. Scope image of a measurement of the load current (up, $2 \mathrm{~A} / \mathrm{div}$ ) and phase voltage (down, $50 \mathrm{~V} / \mathrm{div}$ ), $10 \mathrm{~ms} / \mathrm{div}$.

The two analysis methods used for the simulations are now applied on the experimental results. The control quality of the current and flying capacitor 

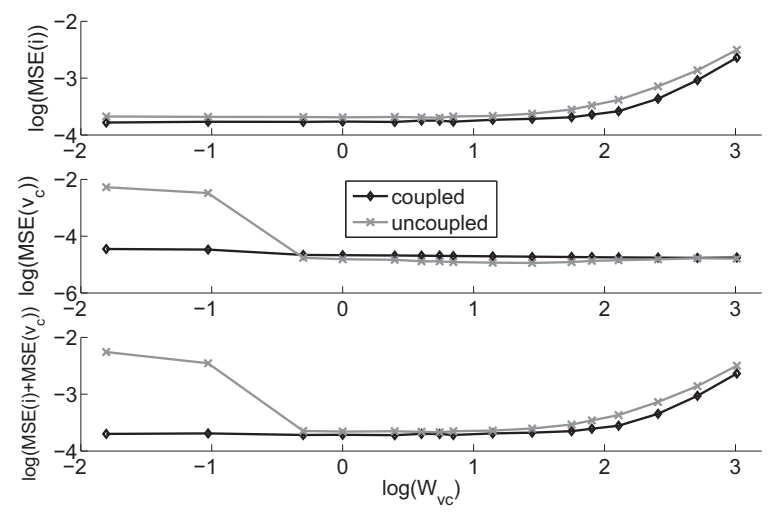

Figure 6. MSE as a function of $W_{v c}$ of (top) output current, (middle) capacitor voltage and (bottom) the sum of the MSE's of current and voltage to define overall control quality using experimental results.

voltages in these experiments was analysed in [18], so here we focus on the voltage quality.

In [18] it is shown that the weight factor range found using simulations corresponds very well with experimental verification. A weight factor value selected using proper simulations will result in a good control quality of the real system. The experimental results of the control quality corresponding with the simulations of figure 2 are depicted in figure 6 , for a phase current amplitude of $4 \mathrm{~A}$. The experimental results correspond very well with the simulations as the same range of good weight factors is obtained. It should be noted that the MSE values itself are different (higher) because of noise in the measurements which are not modelled in the simulations. The current quality in the coupled case is better than in the uncoupled case. For the uncoupled case the quality of the capacitor voltage deteriorates much faster for low weight factors.

The MSE of the output voltage, relative to the $50 \mathrm{~Hz}$ component, is depicted in figure 7. Again the absolute values are higher than in the simulations due to measurement noise, but the behaviour of the MSE of the output voltage is very similar to that of the simulations. The difference between the coupled and uncoupled case is significant.

For high weight factors the MBPC behaves differently in the experiments than in the simulations. In the simulations the intermediate voltage, making use of the flying capacitor, is not used because the MBPC wants to avoid deviation of the capacitor voltage. The "measurements", the input data from the system, in simulation are perfect. A constant

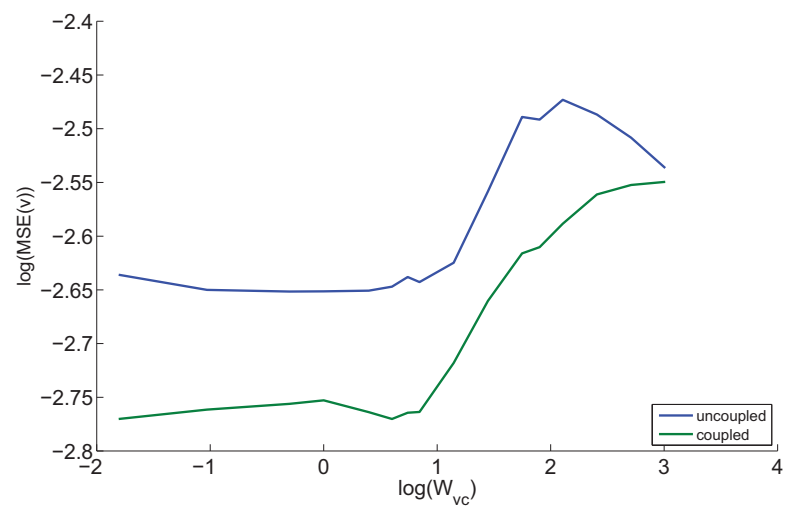

Figure 7. Measurements of MBPC on three-level FC inverter.

voltage stays exactly constant in simulations. In the experiments however, the noise of the measurements of the flying capacitor voltage, although very small, results in a very nervous control when a high weight factor is used. The MBPC is forced to take immediate actions to correct the noisy capacitor voltage measurements. This proofs that the very high weight factors are totally inefficient in practical systems if no extra filters are used, which is not discussed in this paper.

The analysis of the voltage variations in the threephase $\alpha / \beta$-frame is depicted in figure 8 . The full lines show the relative number of updates selecting the same vector (dash-dot lines) or adjacent (dashed lines) and by this complying with the nearest vector selection criterion. The result for the coupled case is significantly higher than for the uncoupled case. The difference, however, is less than for the simulations. This is again due to noise in the measurements and a non-perfect system model for the MPBC.

The decrease of the ratio of nearest vector switchings for high weight factors is less significant than in the simulations. This is again due to the opposite reaction of the MBPC in simulations and in the experimental verification. In simulation it results in skipping the intermediate voltage, in experiment it focuses on the intermediate voltage. Because for high weight factors the flying capacitor terms are most important and there the influence of the starpoint voltage is very small, the behaviour of the MBPC in the coupled and the uncoupled case is almost equal. This is also visible in the MSE values of figure 7 . 


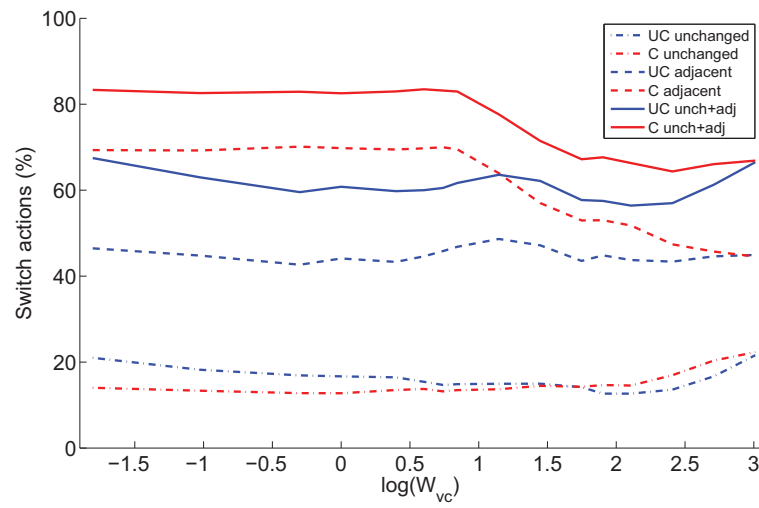

Figure 8. Nearest vector selection: full lines are the sum of the number of unchanged output vectors (dash-dot lines) and the number of transitions to adjacent vector selection (dashed lines) as a function of $W_{v c}$ for the coupled (red) and uncoupled (blue) case using experimental results.

\section{CONCLUSIONS}

MPBC is an attractive option for controlling FC converters. The control quality for two possible cases, the coupled case (with the star-point voltage) and the uncoupled case (neglecting the star-point voltage) was analyzed before. In this paper the voltage quality was analyzed depending on control parameters and model imperfections (star-point voltage). Two ways of analyzing the output voltage quality were introduced. The first is based on the MSE of the deviation of the instantaneous output voltage from the fundamental component of the output voltage. The second is based on the threephase space vector state transition.

The MSE of the output voltage gives a good idea of the quality of the output voltage and the losses it can cause in the load. A clear difference between the coupled and the uncoupled case can be noticed. The experimental verification results in a comparable fluctuation for the weight factor to the simulations. The values itself are higher in the experimental results due to measurement noise and model imperfections.

The analysis of the transitions in the $(\alpha, \beta)$ frame gives us an idea of the cause of the MSE variations. Due to large voltage steps, the MSE value will rise. The best quality will be achieved when only the smallest possible steps are used by the predictive control. Measurement noise and model imperfections can cause the control to react in an inappropriate way, causing deviation from the reference value. This results in large voltage steps to correct the deviation.

With MBPC, the coupled case, where the starpoint voltage is taken into account, always gives better results. The range of $W_{v c}$ resulting in good voltage quality, corresponds with the good range of $W_{v c}$ for the controlled variables (current and flying capacitor voltage). For high weight factors, there is a difference in control reaction between simulation and experiments due to measurement noise and model imperfections. However, the quality analysis gives the same results. It can be concluded that the voltage quality is very sensitive to model imperfections, even more than the influence on the controlled variables. The MBPC is able to control the controlled variables in a relative good way with an imperfect model (uncoupled), but needs more actions for that result causing a worse voltage quality.

After this analysis of the voltage quality of a FC converter controlled with MBPC, in future research the effect of taking the voltage quality as a controlled variable in the cost function will be analyzed.

\section{ACKNOWLEDGEMENT}

This work was supported by the Fund of Scientific Research Flanders (FWO-Flanders) under Project G.0665.06 and by the Interuniversity Attraction Pole under Project (IUAP) P6/21. The work of T.J. Vyncke was supported by a Ph.D. fellowship from the FWO-Flanders.

\section{REFERENCES}

[1] T. Meynard, M. Fadel, and N. Aouda, "Modeling of multilevel converters," Industrial Electronics, IEEE Transactions on, vol. 44, no. 3, pp. 356-364, Jun. 1997.

[2] F. Defay, A. M. Llor, and M. Fadel, "Direct Control Strategy for a Four-Level Three-Phase Flying-Capacitor Inverter," Industrial Electronics, IEEE Transactions on, vol. 57, no. 7, pp. 2240-2248, 2010.

[3] S. S. Fazel, S. Bernet, D. Krug, and K. Jalili, "Design and comparison of $4-\mathrm{kV}$ neutral-point-clamped, flyingcapacitor, and series-connected H-bridge multilevel converters," Industry Applications, IEEE Transactions on, vol. 43, no. 4, pp. 1032-1040, 2007.

[4] J. S. Lai and F. Z. Peng, "Multilevel converters-a new breed of power converters," Industry Applications, IEEE Transactions on, vol. 32, no. 3, pp. 509-517, 2002.

[5] C. Feng, J. Liang, and V. G. Agelidis, "Modified phaseshifted PWM control for flying capacitor multilevel converters," Power Electronics, IEEE Transactions on, vol. 22, no. 1, pp. 178-185, 2007.

[6] H. A. Hotait, A. M. Massoud, S. J. Finney, and B. W. Williams, "Capacitor voltage balancing using redundant states of space vector modulation for five-level diode clamped inverters," Power Electronics, IET, vol. 3, no. 2, pp. 292-313, 2010. 
[7] P. Lezana, R. Aguilera, and D. E. Quevedo, "Model predictive control of an asymmetric flying capacitor converter," Industrial Electronics, IEEE Transactions on, vol. 56, no. 6, pp. 1839-1846, 2009.

[8] S. Thielemans, A. Ruderman, B. Reznikov, and J. A. A. Melkebeek, "Self-precharge for single-leg odd-level multilevel converter," in Power Electronics, Machines and Drives (PEMD 2010), 5th IET International Conference on. IET, 2010, pp. 1-6.

[9] M. F. Escalante, J. C. Vannier, and A. Arzandé, "Flying capacitor multilevel inverters and DTC motor drive applications," Industrial Electronics, IEEE Transactions on, vol. 49, no. 4, pp. 809-815, 2002.

[10] B. P. McGrath and D. G. Holmes, "Analytical modelling of voltage balance dynamics for a flying capacitor multilevel converter," Power Electronics, IEEE Transactions on, vol. 23, no. 2, pp. 543-550, 2008.

[11] A. Ruderman and B. Reznikov, "Five-level single-leg flying capacitor converter voltage balance dynamics analysis," in Industrial Electronics, 2009. IECON '09. 35th Annual Conference of IEEE, 2009, pp. 486-491.

[12] S. Thielemans, A. Ruderman, B. Reznikov, and J. A. A. Melkebeek, "Five-level H-bridge flying capacitor converter voltage balance dynamics analysis," in Industrial Electronics (ISIE), 2010 IEEE International Symposium on. IEEE, pp. 826-831.

[13] R. Wilkinson, T. Meynard, and H. du Toit Mouton, "Natural balance of multicell converters: The two-cell case," Power Electronics, IEEE Transactions on, vol. 21, no. 6, pp. 1649-1657, 2006.
[14] P. Cortes, J. Rodriguez, S. Vazquez, and L. Franquelo, "Predictive control of a three-phase ups inverter using two steps prediction horizon," in Industrial Technology (ICIT), 2010 IEEE International Conference on, 2010, pp. 12831288.

[15] M. Khazraei, H. Sepahvand, K. Corzine, and M. Ferdowsi, "A generalized capacitor voltage balancing scheme for flying capacitor multilevel converters," in Applied Power Electronics Conference and Exposition (APEC), 2010 Twenty-Fifth Annual IEEE, 2010, pp. 58-62.

[16] P. Zanchetta, D. Gerry, V. Monopoli, J. Clare, and P. Wheeler, "Predictive current control for multilevel active rectifiers with reduced switching frequency," Industrial Electronics, IEEE Transactions on, vol. 55, no. 1, pp. 163172, 2008.

[17] J. Rodriguez, B. Wu, M. Rivera, C. Rojas, V. Yaramasu, and A. Wilson, "Predictive current control of threephase two-level four-leg inverter," in Power Electronics and Motion Control Conference (EPE/PEMC), 2010 14th International, 2010, pp. T3-106-T3-110.

[18] T. J. Vyncke, S. Thielemans, M. Jacxsens, and J. A. A. Melkebeek, COMPEL: The International Journal for Computation and Mathematics in Electrical and Electronic Engineering.

[19] A. Ruderman and B. Reznikov, "Pwm power converter voltage quality bounds and their applicability to nonpwm control schemes," in Optimization of Electrical and Electronic Equipment (OPTIM), 2010 12th International Conference on, May 2010, pp. 618-624. 